\title{
Design of Mobile Phone-based Intervention on Health Promotion for the Elderly in Shiraz of Iran
}

\author{
A Asadollahi ${ }^{1,2, *}$ \\ ${ }^{1}$ Department of Healthy Aging, Faculty of Health, Shiraz University of Medical Sciences, Shiraz, Iran \\ ${ }^{2}$ Australian Centre for Quality of Life, Deakin University, Melbourne, Australia \\ ${ }^{*}$ Corresponding author: A Asadollahi, Department of Healthy Aging, Faculty of Health, Shiraz University of Medical Sciences, Shiraz. Iran. E-mail: a.asadollahi@deakin.edu.au
}

Received: 11 Dec 2016

Accepted: 01 Jan 2017

Epub: 23 Feb 2017

Ppub: 15 Jan 2018

\begin{abstract}
Objectives: According to the statistical centre of Iran, a country with 75 million inhabitants in 2014, 6159000 elderly individuals gives it the equivalent of $8.2 \%$ of the total population. In other words, out of every 100 , at least 8 people are elderly. However, the highest and lowest aging population in the 2014 , is from the provinces of Tehran (1.1 million) and Ilam (39 thousand people), respectively. The situation of elderly population in the Fars province, although compared to the national average, is in the lower midtable, however, the province has a main population of elderly. Fars province's population, according to the 2014 census, is 4596658 people, however, the population of the elderly people is equivalent to $8.1 \%$ of the 372,328 older adults. Increasing per capita healthcare would reach more of the older population. Nonetheless, it is proportional to the acceleration of the aging population for comfort and optimal maintenance of the cortex with the necessary planning. The average age of 20 years old, from $1982 \mathrm{until}$ now, added to 78 year old Iranians and life expectancy has grown in 2016. Increasing the average age and increasing health programs is a result of aging. The wave of aging in our society has of course positive and negative consequences. One possible outcome is that many organizations and institutions were not prepared to deal effectively with this phenomenon. Technology and new mobile phone-based can intervene on health promotion, especially regarding the aged community.

Discussion: Mobile phones are the ideal terminal to deliver health services that enhance patients' self-management behaviors in daily life and many mobile phone-based health interventions have been proposed in previous studies. Most interventions enhanced patients' adherence behaviors by facilitating the record of health information and involving the healthcare team. Interventions that record and analyze health data allowed patients to better understand their own condition. In addition, interventions that involve the healthcare team provided patients with professional explanations for the health data and professional advices in improving their self-management behaviors. Some interventions also applied other strategies to motivate patients' self-management behaviors, such as leveraging social influence or utilizing entertainment. Health interventions in previous studies mainly focused on the external support for patients, i.e., support from the devices or from the social environment. However, patients' health beliefs barely changed during the intervention. Interventions that modify patients' health beliefs, such as perceived severity of the condition, or the self-efficacy in maintaining a healthy lifestyle, would also enhance patients' self-management behaviors during chronic condition control. Regarding evaluation of mobile phone-based intervention, mobile phone-based health interventions were proving to be helpful for the control of chronic condition. Empirical data indicated that the number of patients who achieved the goal BP level was 5 times higher when the patients received self-management support by short message services. A web-based intervention using both short message services and Internet improved patients' waist circumference, body weight, and blood pressure significantly during 12 weeks. In another study, the same intervention improved the blood pressure, body weight and, waist circumference of obese patients with hypertension during merely 8 weeks. These studies mainly collected patients' physical measures to indicate the effectiveness of the intervention. There are also studies that discussed the change of patients' internal emotions such as attitude, behavior intention, and subjective norm during the intervention.

Conclusion: Decision makers and health strategic providers in Iran and Shiraz city must attend to the increasing and becoming issues of older people and their old needs into the modern technology as mHealth, mobile phone-based intervention, on the elderly health promotion.
\end{abstract}

Keywords: mHealth; Health Promotion; Elderly People; Health Care 